\title{
New results in low-energy fusion of ${ }^{40} \mathrm{Ca}+{ }^{90,92} \mathrm{Zr}$
}

\author{
A. M. Stefanini, ${ }^{1}$ G. Montagnoli, ${ }^{2}$ H. Esbensen, ${ }^{3}$ P. Čolović, ${ }^{4}$ L. Corradi, ${ }^{1}$ E. Fioretto, ${ }^{1}$ F. Galtarossa,${ }^{1,5}$ A. Goasduff, ${ }^{1}$ \\ J. Grebosz, ${ }^{6}$ F. Haas, ${ }^{7}$ M. Mazzocco, ${ }^{2}$ N. Soić, ${ }^{4}$ E. Strano, ${ }^{2}$ and S. Szilner ${ }^{4}$ \\ ${ }^{1}$ INFN, Laboratori Nazionali di Legnaro, I-35020 Legnaro (Padova), Italy \\ ${ }^{2}$ Dipartimento di Fisica e Astronomia, Università di Padova, and INFN, Sezione di Padova, I-35131 Padova, Italy \\ ${ }^{3}$ Physics Division, Argonne National Laboratory, Argonne, Illinois 60439, USA \\ ${ }^{4}$ Ruđer Bošković Institute, HR-10002 Zagreb, Croatia \\ ${ }^{5}$ Dipartimento di Fisica e Scienze della Terra, Università di Ferrara, I-44121 Ferrara, Italy \\ ${ }^{6}$ Institute of Nuclear Physics, Polish Academy of Sciences, PL 31-342 Cracow, Poland \\ ${ }^{7}$ IPHC, CNRS-IN2P3, Université de Strasbourg, F-67037 Strasbourg Cedex 2, France
}

(Received 7 April 2017; published 7 July 2017)

\begin{abstract}
Background: Near- and sub-barrier fusion of various $\mathrm{Ca}+\mathrm{Zr}$ isotopic combinations have been widely investigated. A recent analysis of ${ }^{40} \mathrm{Ca}+{ }^{96} \mathrm{Zr}$ data has highlighted the importance of couplings to multiphonon excitations and to both neutron and proton transfer channels. Analogous studies of ${ }^{40} \mathrm{Ca}+{ }^{90} \mathrm{Zr}$ tend to exclude any role of transfer couplings. However, the lowest measured cross section for this system is rather high $(840 \mu \mathrm{b})$. A rather complete data set is available for ${ }^{40} \mathrm{Ca}+{ }^{94} \mathrm{Zr}$, while no measurement of ${ }^{40} \mathrm{Ca}+{ }^{92} \mathrm{Zr}$ fusion has been performed in the past.

Purpose: Our aim is to measure the full excitation function of ${ }^{40} \mathrm{Ca}+{ }^{92} \mathrm{Zr}$ near the barrier and to extend downward the existing data on ${ }^{40} \mathrm{Ca}+{ }^{90} \mathrm{Zr}$, in order to estimate the transfer couplings that should be used in coupled-channels calculations of the fusion of these two systems and of ${ }^{40} \mathrm{Ca}+{ }^{94} \mathrm{Zr}$.

Methods: ${ }^{40} \mathrm{Ca}$ beams from the XTU Tandem accelerator of INFN-Laboratori Nazionali di Legnaro were used, bombarding thin metallic ${ }^{90} \mathrm{Zr}\left(50 \mu \mathrm{g} / \mathrm{cm}^{2}\right)$ and ${ }^{92} \mathrm{ZrO}_{2}$ targets (same thickness) enriched to $99.36 \%$ and $98.06 \%$ in masses 90 and 92, respectively. An electrostatic beam deflector allowed the detection of fusion evaporation residues (ER) at very forward angles, and angular distributions of ER were measured.

Results: The excitation function of ${ }^{40} \mathrm{Ca}+{ }^{92} \mathrm{Zr}$ has been measured down to the level of $\simeq 60 \mu \mathrm{b}$. Coupled-channels (CC) calculations using a standard Woods-Saxon (WS) potential and following the line of a previous analysis of ${ }^{40} \mathrm{Ca}+{ }^{96} \mathrm{Zr}$ fusion data give a good account of the new data, as well as of the existing data for ${ }^{40} \mathrm{Ca}+{ }^{94} \mathrm{Zr}$. The previous excitation function of ${ }^{40} \mathrm{Ca}+{ }^{90} \mathrm{Zr}$ has been extended down to $40 \mu \mathrm{b}$.

Conclusions: Transfer couplings play an important role in explaining the fusion data for ${ }^{40} \mathrm{Ca}+{ }^{92} \mathrm{Zr}$ and ${ }^{40} \mathrm{Ca}+{ }^{94} \mathrm{Zr}$. The strength of the pair-transfer coupling is deduced by applying a simple recipe based on the value obtained for ${ }^{40} \mathrm{Ca}+{ }^{96} \mathrm{Zr}$. The logarithmic slopes and the $S$ factors for fusion are reproduced fairly well for all three systems by the CC calculations, and there are no indications of a fusion hindrance at the lowest energies. In contrast, the new data for ${ }^{40} \mathrm{Ca}+{ }^{90} \mathrm{Zr}$ indicate the onset of a fusion hindrance at the lowest energies.
\end{abstract}

DOI: 10.1103/PhysRevC.96.014603

\section{INTRODUCTION}

A wide range of phenomena have been observed in nearand sub-barrier heavy-ion fusion reactions, and couplings to nucleon transfer channels have been recognized to enhance significantly the cross sections for several systems [1]. The first suggestion of Broglia et al. [2] was that two-neutron transfer with $Q>0$ should enhance sub-barrier fusion, following the results of the early experiments of Beckerman et al. [3] on various $\mathrm{Ni}+\mathrm{Ni}$ systems. Nucleon transfer effects should show up rather clearly down to energies well below the barrier, just where the fusion hindrance is expected to set in [1]. Therefore, the low-energy fusion cross sections may be influenced by the concurring contributions of hindrance and enhancement, and disentangling the underlying dynamics can be problematic.

The various $\mathrm{Ca}+\mathrm{Zr}$ systems are very significant in this respect. The sub-barrier fusion excitation function of ${ }^{40} \mathrm{Ca}+$ ${ }^{96} \mathrm{Zr}$ was recently measured down to cross sections $\simeq 2.4 \mu \mathrm{b}$ [4], i.e., two orders of magnitude smaller than obtained in a previous experiment [5]. The sub-barrier fusion of this system was found to be greatly enhanced with respect to ${ }^{40} \mathrm{Ca}+{ }^{90} \mathrm{Zr}$, calling for additional couplings to transfer channels.

The recent coupled channels (CC) analysis [6] of the excitation function of ${ }^{40} \mathrm{Ca}+{ }^{96} \mathrm{Zr}$ used a WS potential and included explicitly, besides multiphonon excitations, one- and two-nucleon $Q>0$ transfer channels, with coupling strengths calibrated to reproduce the measured neutron transfer data [7]. Such transfer couplings bring significant cross section enhancements, even at the level of a few $\mu \mathrm{b}$. However, the couplings to neutron transfer are not strong enough to explain the fusion data at subbarrier energies. It is necessary also to consider the influence of proton stripping channels with positive $Q$ values if one wants to develop a good account of the fusion data [6].

Calculations concerning ${ }^{40} \mathrm{Ca}+{ }^{90} \mathrm{Zr}$ [8] tend to exclude any role of transfer couplings. However, the lowest measured cross section for this system is rather large $(840 \mu \mathrm{b})$. CC calculations that use a standard Woods-Saxon potential give a data fit essentially as good as that obtained with the 
M3Y + repulsion potential [8,9]. The reason is that the lowest measured cross section is such that the expected fusion hindrance at very low energies has not yet set in. It is therefore of great interest to continue the measurements to much smaller cross sections in order to determine whether the fusion hindrance phenomenon exists in these systems.

In ${ }^{40} \mathrm{Ca}+{ }^{96} \mathrm{Zr}$ no indication of a fusion hindrance shows up either, down to the $\mu \mathrm{b}$ range, and locating the hindrance threshold in this system, if any, would require challenging measurements of very small cross sections. The suggested interpretation of this surprising behavior [8] has been that, since the $Q$ values for nucleon transfer are large and positive in ${ }^{40} \mathrm{Ca}+{ }^{96} \mathrm{Zr}$, the valence nucleons can flow more freely from one nucleus to the other without being hindered by Pauli blocking. In systems where the transfer $Q$ values are large and negative, the Pauli blocking will result in a repulsion between the reacting nuclei and lead to a fusion hindrance at very low energies [10]. We note that a similar repulsion caused by the nuclear incompressibility was previously used to explain the fusion hindrance phenomenon [9], but the connection between the two mechanisms has not yet been fully explored.

To our knowledge, no measurement of ${ }^{40} \mathrm{Ca}+{ }^{92} \mathrm{Zr}$ fusion cross sections has been performed in the past. The transfer $Q$ values for this systems are smaller than in ${ }^{40} \mathrm{Ca}+{ }^{96} \mathrm{Zr}$ reactions and it is therefore of interest to find out how large is the influence of couplings to transfer channels. The excitation function of ${ }^{40} \mathrm{Ca}+{ }^{94} \mathrm{Zr}$ already exists [11] and it will also be analysed in this work. It is quite similar to the ${ }^{40} \mathrm{Ca}+{ }^{96} \mathrm{Zr}$ fusion data, consistent with the fact that the transfer $Q$ values for the two systems are positive and similar in magnitude.

In this article we have extended the existing data on ${ }^{40} \mathrm{Ca}+{ }^{90} \mathrm{Zr}$ [5] downward by a factor of 20 . We also present the results of measurements of the full excitation function of ${ }^{40} \mathrm{Ca}+{ }^{92} \mathrm{Zr}$ at energies near and below the Coulomb barrier. Section II describes the experimental setup and shows the results that will be analysed in Sec. III using CC calculations. A final discussion will follow in Sec. V, and the conclusions are summarized in Sec. V.

\section{EXPERIMENT}

${ }^{40} \mathrm{Ca}$ beams in the energy range $128-155 \mathrm{MeV}$, with intensities $\simeq 4-7$ p nA were provided by the XTU Tandem accelerator of the Laboratori Nazionali di Legnaro of INFN. The ${ }^{90} \mathrm{Zr}\left({ }^{92} \mathrm{Zr}\right)$ targets were installed in a sliding seal scattering chamber, and consisted of $50 \mu \mathrm{g} / \mathrm{cm}^{2} \mathrm{Zr}\left(\mathrm{ZrO}_{2}\right)$ evaporations on 15 (20) $\mu \mathrm{g} / \mathrm{cm}^{2}$ carbon backings facing the beam.

The isotopic composition of the ${ }^{90} \mathrm{Zr}$ target was $99,36 \%$, $0.30 \%, 0.17 \%, 0.12 \%$, and $0.04 \%$ for ${ }^{90,91,92,94,96} \mathrm{Zr}$, respectively. The ${ }^{92} \mathrm{Zr}$ target was composed of $98.06 \%, 0.97 \%$, $0.51 \%, 0.41 \%$, and $0.05 \%{ }^{92,90,91,94,96} \mathrm{Zr}$, respectively. In order to take account of these isotopic contaminations, we applied the following procedure: first, the observed fusion yields of ${ }^{40} \mathrm{Ca}+{ }^{92} \mathrm{Zr}$ were corrected using the already measured excitation functions of ${ }^{40} \mathrm{Ca}+{ }^{94,96} \mathrm{Zr}$. The other two systems ${ }^{40} \mathrm{Ca}+{ }^{90,91} \mathrm{Zr}$ do not bring significant contributions, because their barriers are higher by about 1 and $2 \mathrm{MeV}$ in the laboratory system.
Then the raw data of ${ }^{40} \mathrm{Ca}+{ }^{90} \mathrm{Zr}$ were corrected using the resulting values of ${ }^{40} \mathrm{Ca}+{ }^{92} \mathrm{Zr}$ and the measured excitation functions of ${ }^{40} \mathrm{Ca}+{ }^{94,96} \mathrm{Zr}$. For the system ${ }^{40} \mathrm{Ca}+{ }^{91} \mathrm{Zr}$ we have used interpolated cross sections. The isotopic corrections were negligible near and above the barrier for both targets but increased at lower energies.

A further source of uncertainty comes from a ${ }^{88} \mathrm{Sr}$ impurity $(<100 \mathrm{ppm}$, as stated by the company producing the enriched isotopes). Other very small elemental impurities with lower $Z$ (not observed) would have been anyway separated out by our $\Delta E-E$ telescope.

The overall consequence of the isotopic and elemental contaminants was that we could not measure fusion cross sections lower than $\simeq 40-60 \mu \mathrm{b}$. The beam energy loss in the carbon backing and the zirconium targets was taken into account in the data analysis.

Four silicon detectors were used for beam control and normalization between the different runs by measuring the Rutherford scattering from the target. They were placed above and below and to the left and right of the beam at the same scattering angle $\theta_{\text {lab }}=23.2^{\circ}$.

The ER were detected by using the setup based on an electrostatic beam deflector, that is described in some detail in Ref. [12]. Following the separation from the beam, the ER entered a telescope consisting of two micro-channel plate detectors (MCP) and a transverse-field ionization chamber IC giving an energy loss $(\Delta E)$ signal, and were finally stopped in a circular $600 \mathrm{~mm}^{2}$ silicon detector placed in the same gas $\left(\mathrm{CH}_{4}\right)$ volume. The silicon detector provided the residual energy $E_{R}$, as well as the start signal used for the times of flight TOF from each MCP, and triggered the data acquisition. The total length of the detector telescope was $\simeq 105 \mathrm{~cm}$ with a geometrical solid angle of the whole setup of $\Delta \Omega=0.036 \pm 0.003 \mathrm{msr}$ (determined by the silicon detector size).

The fusion yields were measured at $\theta_{\text {lab }}=2^{\circ}$ with respect to the beam direction. The ER angular distribution was measured at $E_{\text {lab }}=152 \mathrm{MeV}$ in the range $-7^{\circ}$ to $+6^{\circ}$, for both systems, in steps of $1^{\circ}$. The solid angle of all detectors, including the $E-\Delta E$-TOF telescope, was determined by placing an $\alpha$ source at the target position. Total fusion cross sections result from normalizing to the values obtained at a few energies above the barrier in the previous measurements for ${ }^{40} \mathrm{Ca}+{ }^{90} \mathrm{Zr}$ [5].

Relative errors of the cross sections are essentially determined by statistical uncertainties which do not exceed 2-3\% near and above the barrier, but become much larger, and dominate, at low energies where few fusion events could be detected. Systematic errors on the absolute cross section scale are estimated $\pm 7-8 \%$ (see Ref. [12]).

The new cross sections for the fusion of ${ }^{40} \mathrm{Ca}$ and ${ }^{90,92} \mathrm{Zr}$ that we have measured are compared in Fig. 1 (solid diamonds) to previous data for ${ }^{40} \mathrm{Ca}+{ }^{90} \mathrm{Zr}$ [5] and to the results of $\mathrm{CC}$ calculations that are discussed in the next section. The Ch-29 calculations are the most complete calculations that are based entirely on the multiphonon excitations. They provide a fairly good description of the ${ }^{40} \mathrm{Ca}+{ }^{90} \mathrm{Zr}$ data, whereas the discrepancy between the $\mathrm{Ch}-29$ calculation and the ${ }^{40} \mathrm{Ca}+{ }^{92} \mathrm{Zr}$ data is very large at low energies. The discrepancy is resolved in Ch-87 calculations by including couplings to 

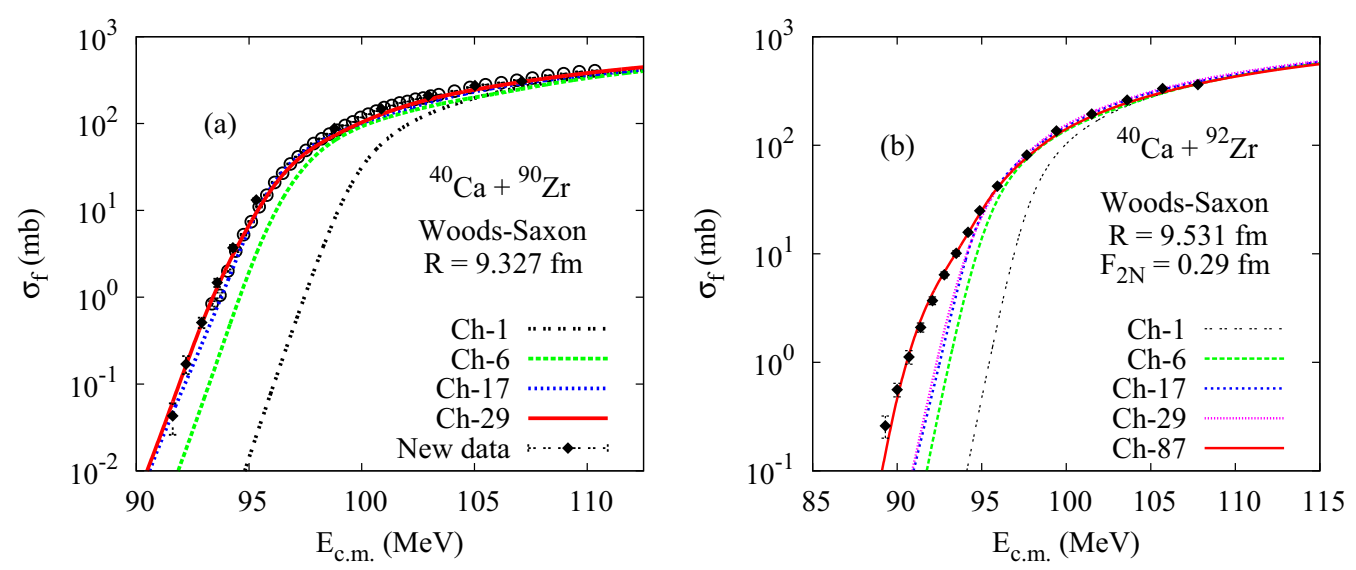

FIG. 1. Excitation functions of ${ }^{40} \mathrm{Ca}+{ }^{90} \mathrm{Zr}$ (a) and ${ }^{40} \mathrm{Ca}+{ }^{92} \mathrm{Zr}$ (b) are compared to the $\mathrm{CC}$ calculations described in the text. The solid diamonds are the new data, whereas the open circles in (a) are the ${ }^{40} \mathrm{Ca}+{ }^{90} \mathrm{Zr}$ data by Timmers et al. [5].

one- and two-nucleon transfer reactions, as explained in the next section.

\section{COUPLED-CHANNELS CALCULATIONS}

The coupled-channels (CC) calculations we have performed use the same formalism that was used in Refs. [6,8] to analyze the fusion reactions between different $\mathrm{Ca}$ and $\mathrm{Zr}$ isotopes. The formalism is based on the so-called rotating frame [13,14] or isocentrifugal [15] approximation, which simplifies the calculations by reducing the number of channels considerably.

The calculations we have performed are exploratory and by no means complete. They are based on our past experience obtained, for example, in Refs. [6,8].

The fusion data for ${ }^{40} \mathrm{Ca}+{ }^{90,92,94,96} \mathrm{Zr}$ are analyzed in this work by $\mathrm{CC}$ calculations that use a standard Woods-Saxon potential [16] and include couplings of up to three-phonon excitations. The couplings to multiphonon excitations are similar to those that were used in Ref. [8] to explain the ${ }^{40} \mathrm{Ca}+{ }^{90} \mathrm{Zr}$ fusion data of Timmers et al. [5]. The fit to the data was fairly good but not perfect, primarily because the data exceeded the calculations at high energies. We will discuss this problem later on.

The analysis of ${ }^{40} \mathrm{Ca}+{ }^{92,94,96} \mathrm{Zr}$ fusion data will include the same kind of couplings to multiphonon excitations and in addition the couplings to one- and two-nucleon transfer reactions. The calculations are similar to those we recently performed for ${ }^{40} \mathrm{Ca}+{ }^{96} \mathrm{Zr}$ [6]. In that work the couplings to one- and two-neutron transfer channels with positive $Q$ values were first calibrated so that the existing neutron transfer data [7] were reproduced. The resulting fusion cross section did not reproduce the data so well. After realizing that couplings to proton transfer channels with positive $Q$ values could also affect the calculated fusion cross section, a simple estimate was made by multiplying the one- and two-neutron transfer strengths by $\sqrt{2}$. The resulting fusion cross sections turned out to be in excellent agreement with the ${ }^{40} \mathrm{Ca}+{ }^{96} \mathrm{Zr}$ fusion data [6].

Inspired by the successful description of the fusion of ${ }^{40} \mathrm{Ca}+{ }^{96} \mathrm{Zr}$ in Ref. [6], we shall therefore try to estimate the strengths of the transfer couplings one should use in CC calculations of the fusion of ${ }^{40} \mathrm{Ca}$ with other $\mathrm{Zr}$ isotopes. The transfer couplings are expected to have a large effect on fusion because the effective $Q$ values shown in Table I are large and positive in most cases. However, the $Q$ values for the neutron and one-proton transfers are negative in ${ }^{40} \mathrm{Ca}+{ }^{90} \mathrm{Zr}$ reactions, and the couplings to transfer reactions are therefore not expected to have a large effect on fusion for this system.

\section{A. Excitation channels}

Multiphonon calculations with up to three-phonon excitations have been performed. The basic nuclear structure input is shown in Table II. Besides the $2^{+}$state, the $3^{-}$and $5^{-}$ excitations in ${ }^{90} \mathrm{Zr}$ are combined into one effective $3^{-}$state so that the calculations for the different $\mathrm{Zr}$ isotopes become similar but the excitation energies and strengths are different. In ${ }^{40} \mathrm{Ca}$ we consider the excitations of the $2^{+}, 3^{-}$, and $5^{-}$states explicitly. The calculation that includes the elastic channel and the one-phonon excitation of the states mentioned above has six channels and is therefore identified as a "Ch-6" calculation.

The multiphonon excitations we consider are described in the harmonic approximation. Since the number of channels can easily become very large and difficult to handle, it is important to eliminate some of the weaker channels and restrict the number of channels with large excitation energies. For example, the quadrupole excitations of all of the nuclei shown in Table II are rather weak, and we will therefore ignore the explicit two- and three-phonon excitations of these states. We

TABLE I. Effective $Q$ values (in $\mathrm{MeV}$ ) for the most favorable one- and two-nucleon transfer reactions in ${ }^{40} \mathrm{Ca}+{ }^{90,92,94,96} \mathrm{Zr}$ collisions, and the adopted strength $F_{2 N}$ of the pair transfer.

\begin{tabular}{lccccc}
\hline \hline System & $Q_{1 n}$ & $Q_{2 n}$ & $Q_{1 p}$ & $Q_{2 p}$ & $F_{2 N}(\mathrm{fm})$ \\
\hline${ }^{40} \mathrm{Ca}+{ }^{90} \mathrm{Zr}$ & -3.50 & -1.25 & -0.73 & +3.05 & 0 \\
${ }^{40} \mathrm{Ca}+{ }^{92} \mathrm{Zr}$ & -0.17 & 4.22 & +0.16 & +4.95 & 0.290 \\
${ }^{40} \mathrm{Ca}+{ }^{94} \mathrm{Zr}$ & +0.25 & +5.09 & +0.91 & +6.50 & 0.324 \\
${ }^{40} \mathrm{Ca}+{ }^{96} \mathrm{Zr}$ & +0.61 & +5.73 & +1.55 & +7.63 & 0.355 \\
\hline \hline
\end{tabular}


TABLE II. Adopted structure of the excited states in ${ }^{40} \mathrm{Ca}$ [17], and ${ }^{90,92,94,96} \mathrm{Zr}[18,19]$. The Coulomb and nuclear coupling strengths $\sigma_{\lambda}$ are determined by the associated $\beta_{\lambda}$ values through the relation $\sigma_{\lambda}=\beta_{\lambda} R_{0} / \sqrt{4 \pi}$, where $R_{0}=1.2 A^{1 / 3}$.

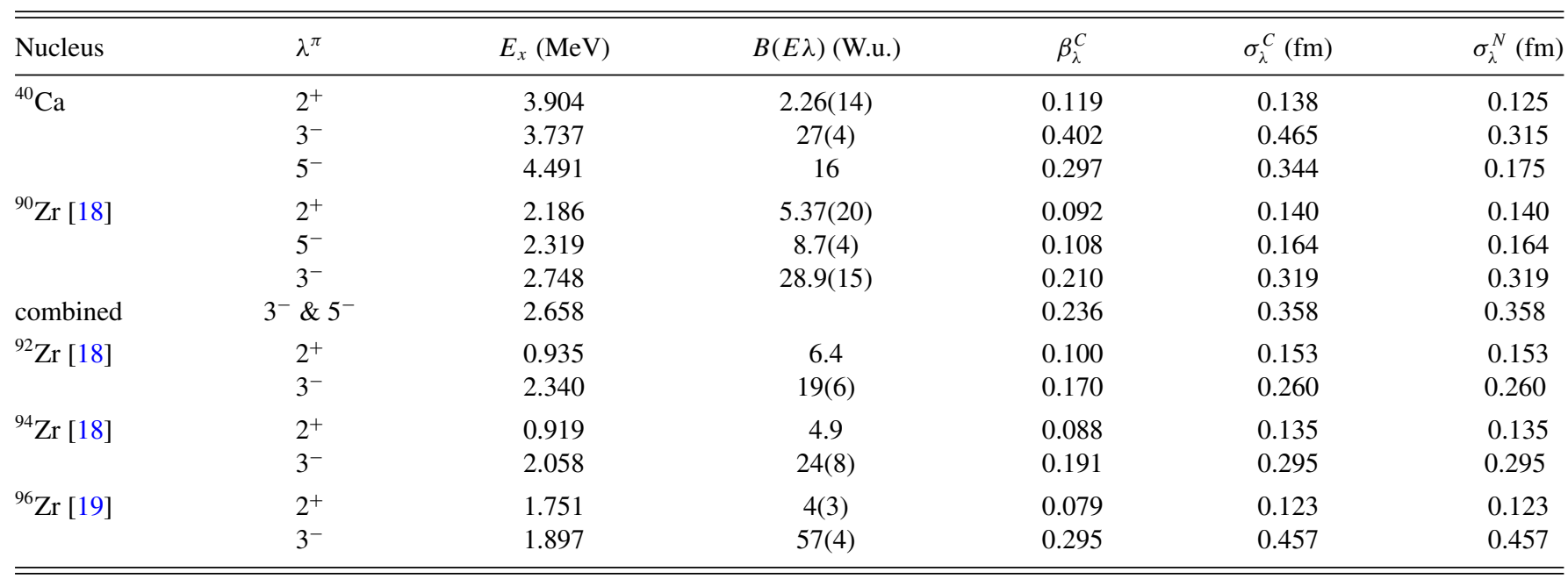

will also ignore the two- and three-phonon excitations of the $3^{-}$and $5^{-}$states in ${ }^{40} \mathrm{Ca}$ because their excitation energies are relatively large. Their effect would mainly be an adiabatic renormalization of the ion-ion potential [20], which we will ignore because it can be compensated or simulated by adjusting the radius of the WS potential.

The only multiphonon excitations of the same state we consider is the two- and three-phonon excitation of the $3^{-}$ state in the $\mathrm{Zr}$ isotopes. The reason is that these states played an important role in explaining the barrier distribution for the fusion of ${ }^{48} \mathrm{Ca}+{ }^{96} \mathrm{Zr}[8,21]$ and also for the fusion of ${ }^{40} \mathrm{Ca}+{ }^{96} \mathrm{Zr}$ [6]. With these restrictions a typical calculation with up to two-phonon excitations is a Ch-17 calculation. It consists of the six zero- and one-phonon channels, the explicit two-phonon excitation of the $3^{-}$state in the $\mathrm{Zr}$ isotope, and the $4+3+2+1=10$ mutual excitations of two different one-phonon states, with a total of 17 channels.

The number of three-phonon excitations that consist of three different one-phonon states is 10 . Combined with the Ch-17 two-phonon calculation mentioned above, the explicit three-phonon excitation of the $3^{-}$state in the $\mathrm{Zr}$ isotopes, and the four excitations that consist of the two-phonon excitation of the $3^{-}$state in $\mathrm{Zr}$ and a different one-phonon state, we end up with a total of 32 channels. This number was reduced to 28 in the $\mathrm{CC}$ calculations for ${ }^{40} \mathrm{Ca}+{ }^{96} \mathrm{Zr}$ by imposing a maximum excitation energy cutoff at $10 \mathrm{MeV}$ [6]. The same cutoff in the reactions of ${ }^{40} \mathrm{Ca}+{ }^{92,94} \mathrm{Zr}$ results in 29 channels. For consistency we also perform $\mathrm{Ch}-29$ coupled-channels calculations for the fusion of ${ }^{40} \mathrm{Ca}+{ }^{90} \mathrm{Zr}$ using the same excitation channels as in the reactions of ${ }^{40} \mathrm{Ca}+{ }^{92,94} \mathrm{Zr}$. This requires an excitation energy cutoff at $10.6 \mathrm{MeV}$.

The results of the calculations for ${ }^{40} \mathrm{Ca}+{ }^{90,92} \mathrm{Zr}$ are shown in Fig. 1. The calculations show in increasing order the results of the no-coupling $\mathrm{Ch}-1$ calculations, the $\mathrm{Ch}-6$ one-phonon, the Ch-17 two-phonon, and the $\mathrm{Ch}-29$ three-phonon calculations described above. It is seen that the $\mathrm{Ch}-29$ calculation provides a good description of the ${ }^{40} \mathrm{Ca}+{ }^{90} \mathrm{Zr}$ data, whereas there is a large discrepancy with the ${ }^{40} \mathrm{Ca}+{ }^{92} \mathrm{Zr}$ data at low energy.

The results of the analysis of the data for the two systems based on Ch-29 calculations are shown in Table III. In each case the radius of the WS potential was adjusted to optimize the fit to the data. While the $\chi^{2} / N$ of the fit is not unreasonable for ${ }^{40} \mathrm{Ca}+{ }^{90} \mathrm{Zr}$, it is very poor for ${ }^{40} \mathrm{Ca}+{ }^{92} \mathrm{Zr}$. The discrepancy with the data is resolved as explained below by including couplings to one- and two-nucleon transfer reactions.

\section{B. Transfer channels}

The CC calculations we have performed with up to threephonon excitations have either 28 or 29 channels as explained

TABLE III. The parameters of the Woods-Saxon ion-ion potential that provide the best fit to the data in Ch-29 and Ch-87 calculations. The strength of the pair-transfer coupling, $F_{2 N}$, is listed in the second to last column. The last column shows the $\chi^{2} / N$ which includes a $5 \%$ or $7 \%$ systematic error, as indicated in the first column. The calculations labeled with a $\left(^{*}\right)$ are adopted as most realistic ones (see text).

\begin{tabular}{|c|c|c|c|c|c|c|c|}
\hline Data & $V_{0}(\mathrm{MeV})$ & $R(\mathrm{fm})$ & $a(\mathrm{fm})$ & $V_{C B}$ & Calc & $F_{2 N}(\mathrm{fm})$ & $\chi^{2} / N$ \\
\hline${ }^{40} \mathrm{Ca}+{ }^{92} \mathrm{Zr} 5 \%$ & 74.24 & 9.586 & 0.672 & 96.81 & Ch-29 & 0 & 16.5 \\
\hline${ }^{40} \mathrm{Ca}+{ }^{92} \mathrm{Zr} 5 \%$ & 75.28 & 9.521 & 0.672 & 97.42 & Ch-87 & 0.355 & 2.62 \\
\hline${ }^{40} \mathrm{Ca}+{ }^{94} \mathrm{Zr}[11] 5 \%$ & 73.73 & 9.605 & 0.672 & 96.59 & $\mathrm{Ch}-87(*)$ & 0.324 & 0.76 \\
\hline${ }^{40} \mathrm{Ca}+{ }^{94} \mathrm{Zr}[11] 5 \%$ & 73.80 & 9.60 & 0.672 & 96.64 & Ch-87 & 0.355 & 0.38 \\
\hline
\end{tabular}


above. When one- and two-nucleon transfer reactions are also included, we assume that excitations and transfers are independent degrees of freedom [6]. The calculations will therefore have either $3 \times 28=84$ or $3 \times 29=87$ channels, and they are referred to as Ch-84 and Ch- 87 calculations, respectively.

The one- and two-neutron transfer strengths that were determined in Ref. [6] by analyzing the existing transfer data [7] were multiplied with the factor $\sqrt{2}$ in order to simulate the combined effect of neutron and proton transfers in ${ }^{40} \mathrm{Ca}+{ }^{96} \mathrm{Zr}$ reactions. In that work a very good fit to the fusion data was achieved in Ch-84 calculations. The parameters of the best calculation are shown in last line of Table III; the best $\chi^{2} / N=0.65$ is extremely good. It turned out that the calculated one- and two-nucleon transfer cross sections were in reasonable agreement with the data [7], which further justifies the adopted $\sqrt{2}$ scaling factor.

We have performed a similar analysis of the existing data for ${ }^{40} \mathrm{Ca}+{ }^{94} \mathrm{Zr}$ [11] and the new ${ }^{40} \mathrm{Ca}+{ }^{92} \mathrm{Zr}$ data. The full calculations include the 29 excitation channels mentioned earlier which combined with one- and two-nucleon transfer reactions results in $\mathrm{Ch}-87$ calculations. The only changes that were made in comparison to the calculations for ${ }^{40} \mathrm{Ca}+{ }^{96} \mathrm{Zr}$ [6] were the energies and strengths of the target excitations, the effective $Q$ values for one- and two-nucleon transfer, and the occupation of the $d_{5 / 2}$ neutron orbit in the $\mathrm{Zr}$ isotope. The effective $Q$ values for one-nucleon transfer shown in Table I were set to the values for the one-neutron transfer in the CC calculations.

The ground state $Q$ values for pair-transfer are very large and positive in most of the systems considered here (see Table I). They were set to $1 \mathrm{MeV}$ in the $\mathrm{CC}$ calculations, as in our recent analyses of $\mathrm{Ca}+\mathrm{Zr}$ systems $[6,8]$, because this value is close to the optimum $Q$ value. Indeed the experimental $Q$-value distribution (when known) is broad and peaks near the optimum value, while the cross section for the ground-state to ground-state transition is small, when the associated $Q$ value is large and positive.

Since the pair transfer is represented in our calculations by one effective channel (apart from vibrational excitations), it is best to choose an effective $Q$ value for that channel which is close to the optimum value. In our previous work $[4,8]$ we found that an effective $Q$ value of $+1 \mathrm{MeV}$ and an adjusted pair-transfer strength gave a good description of the transfer data in ${ }^{40} \mathrm{Ca}+{ }^{96} \mathrm{Zr}$ reactions.

It would be very misleading to use the ground-state to ground-state $Q$ value in our simplified calculations because it would require a very unrealistic and large pair-transfer strength to reproduce the measured transfer cross section. Consequently, the influence on fusion would be misunderstood.

The strength of the direct two-nucleon transfer coupling was first set to $F_{2 N}=0.355 \mathrm{fm}$, which is the value that was determined in the analysis of the ${ }^{40} \mathrm{Ca}+{ }^{96} \mathrm{Zr}$ fusion data [6]. A small adjustment of the radius of the WS potential was made in order to optimize the fit to the data. The parameters of the best $\mathrm{Ch}-87$ calculation are shown in Table III. The $\chi^{2} / N=0.38$ obtained for ${ }^{40} \mathrm{Ca}+{ }^{94} \mathrm{Zr}$ is an extremely small value in view of the small systematic error of $5 \%$ that was used in the analysis. The best fit to the ${ }^{40} \mathrm{Ca}+{ }^{92} \mathrm{Zr}$ fusion data, obtained in $\mathrm{Ch}-87$ calculations with the same pair-transfer strength, $F_{2 N}=0.355 \mathrm{fm}$, has a $\chi^{2} / N=2.62$. The fit is not perfect but it is clearly much better than the value $\chi^{2} / N=16.5$ that was obtained in the $\mathrm{Ch}-29$ calculations. In the section below we investigate the sensitivity to the pair-transfer strength and try to determine a more realistic value.

\section{Adjusting the pair transfer}

The strength of the direct two-neutron transfer in ${ }^{40} \mathrm{Ca}+$ ${ }^{A} \mathrm{Zr}$ reactions is expected to be reduced with a decreasing mass number $A$ of the $\mathrm{Zr}$ isotope, simply because the number of valence neutrons is reduced. The strength of the direct two-proton transfer, on the other hand, is not expected to change dramatically. Assuming as we did in Ref. [6] that the contributions to the total two-nucleon transfer strength from protons and neutrons are the same in ${ }^{40} \mathrm{Ca}+{ }^{96} \mathrm{Zr}$ reactions, we can therefore estimate the total two-nucleon transfer strength for the other $\mathrm{Zr}$ isotopes as follows:

$$
F_{2 N}(A)=\sqrt{1 / 2+1 / 2 *(A-90) / 6} \times 0.355 \mathrm{fm},
$$

where $F_{2 N}=0.355 \mathrm{fm}$ is the pair-transfer strength for ${ }^{40} \mathrm{Ca}+$ ${ }^{96} \mathrm{Zr}$ that was determined in Ref. [6]. The first $1 / 2$ in this expression represents the strength of the direct two-proton transfer. The second $1 / 2$ is weighted by the number of valence neutrons in the nucleus ${ }^{A} \mathrm{Zr}$ so that we get the correct strength for $A=96$. This estimate gives the pair-transfer strength $F_{2 N}=0.324 \mathrm{fm}$ for $A=94$, and $F_{2 N}=0.290$ fm for $A=92$. These are the values we adopt in the following CC calculations.

The $\chi^{2} / N$ we obtain with the estimated pair-transfer strengths are also shown in Table III. It is seen that the strength $F_{2 N}=0.290 \mathrm{fm}$ for ${ }^{40} \mathrm{Ca}+{ }^{92} \mathrm{Zr}$ provides an excellent fit to the data. The fit is much better than the result without any pair transfer, and it is also better that the result with the strength $F_{2 N}=0.355 \mathrm{fm}$. The estimated pair-transfer strength for ${ }^{40} \mathrm{Ca}+{ }^{94} \mathrm{Zr}, F_{2 N}=0.324 \mathrm{fm}$, produces a good fit to the data but the $\chi^{2} / N$ is twice the value obtained with the strength $F_{2 N}=0.355 \mathrm{fm}$. It is therefore not so clear which strength is the most realistic and whether Eq. (1) makes a reliable prediction.

In the following we adopt the calculations labeled with a (*) in Table III as the most realistic calculations we have performed. They are compared in Fig. 2 to the data for the four ${ }^{40} \mathrm{Ca}+{ }^{A} \mathrm{Zr}$ systems. The logarithmic plot in the left panel (a) shows that the calculations provide a fairly consistent description of all of the data at sub-barrier energies. The linear plot in the right panel (b) shows that the calculations in most cases reproduce the data fairly well at high energies. The largest discrepancy is observed as mentioned earlier in the case of ${ }^{40} \mathrm{Ca}+{ }^{90} \mathrm{Zr}$, where the data exceed the calculation substantially.

The comparisons made in Fig. 2 and Table III show that the largest discrepancy between calculations and data occur for the system ${ }^{40} \mathrm{Ca}+{ }^{90} \mathrm{Zr}$. Since the $\mathrm{Ch}-29$ calculation presented in this case does not include any effect of transfer, one might suspect that this is the reason why the discrepancy is large. However, this is most likely not the reason. A characteristic feature of the couplings to transfer channels is that they 

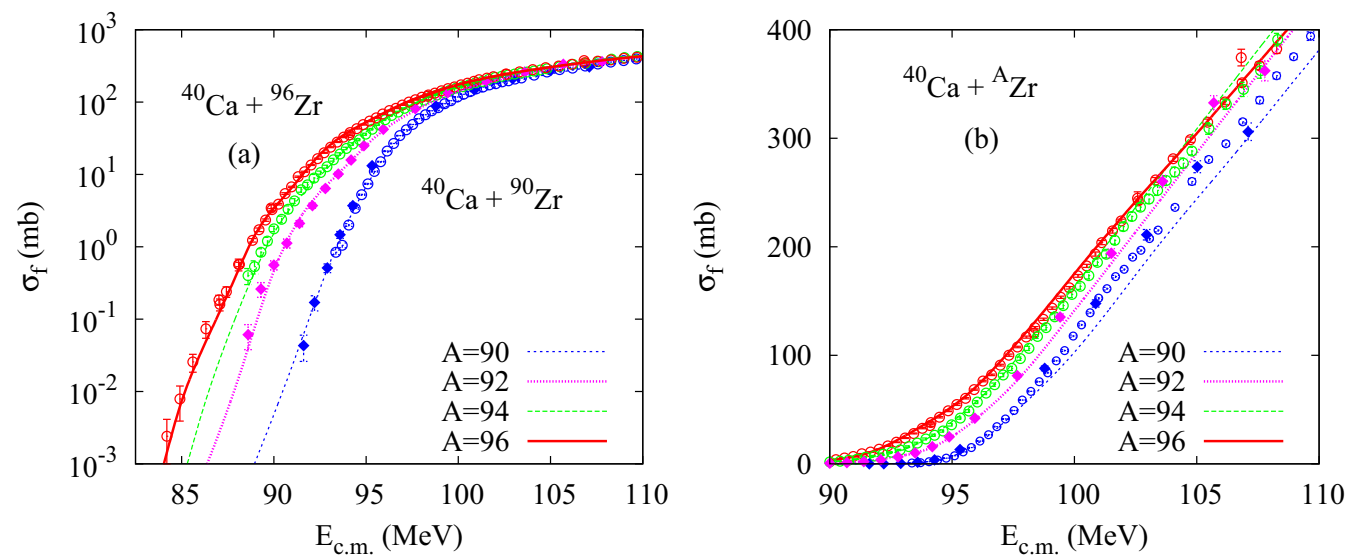

FIG. 2. Measured cross sections for the fusion of ${ }^{40} \mathrm{Ca}+{ }^{90,96} \mathrm{Zr}[4,5],{ }^{40} \mathrm{Ca}+{ }^{94} \mathrm{Zr}$ [11], and the new data for ${ }^{40} \mathrm{Ca}+{ }^{90,92} \mathrm{Zr}$ (solid diamonds) are compared to $\mathrm{CC}$ calculations in a logarithmic (a) and a linear (b) plot, respectively. The parameters of the calculations are indicated with a (*) in Table III.

enhance the fusion cross section at low energies and reduce it at high energies.

This can be seen in Fig. 4 of our previous analysis of the ${ }^{40} \mathrm{Ca}+{ }^{96} \mathrm{Zr}$ fusion data [6]. By comparing in that figure the $\mathrm{Ch}-84$ calculation (with transfer) to the $\mathrm{Ch}-28$ calculation
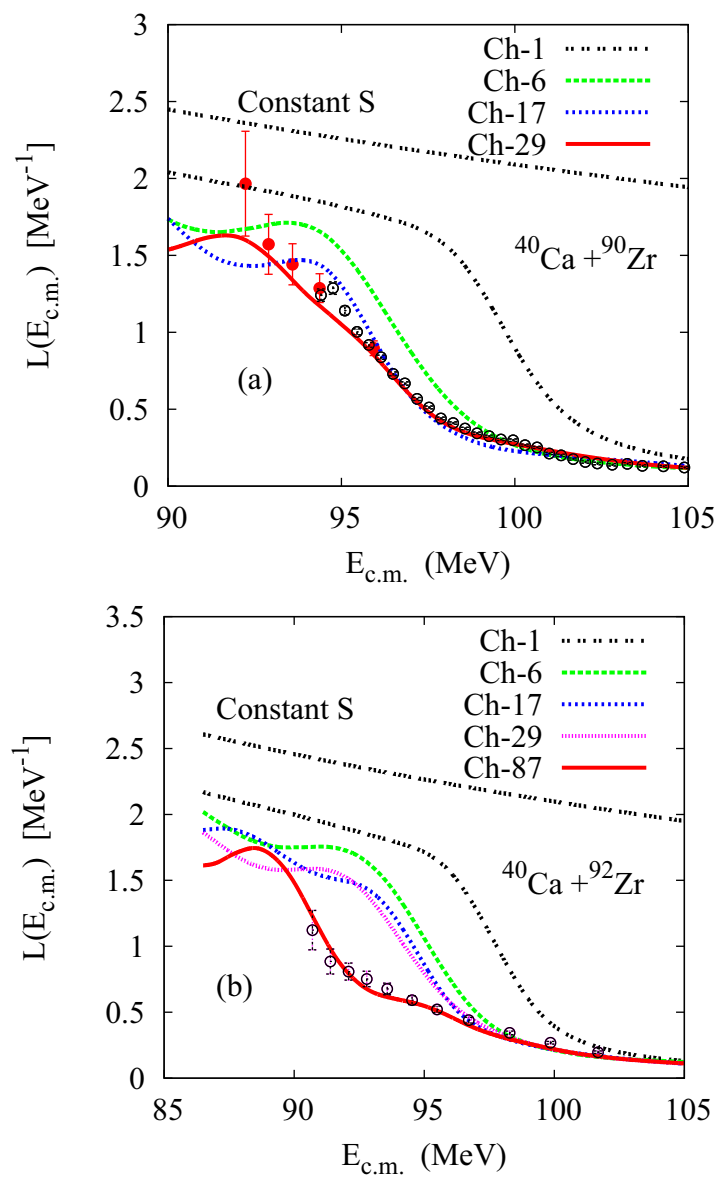

FIG. 3. Logarithmic slopes of the excitation function of ${ }^{40} \mathrm{Ca}+$ ${ }^{90} \mathrm{Zr}$ (a) and ${ }^{40} \mathrm{Ca}+{ }^{92} \mathrm{Zr}$ (b) are compared to $\mathrm{CC}$ calculations. The solid (red) points in (a) are the results of the new measurements for ${ }^{40} \mathrm{Ca}+{ }^{90} \mathrm{Zr}$. (without transfer) it is seen that transfer enhances the fusion cross section at low energies [Fig. 4(a)] and reduces it at high energies [Fig. 4(b)]. In fact, these features are part of a general trend that was discussed in Sec. II E of Ref. [1] and has been observed, for example, in the fusion of $\mathrm{Ni}+\mathrm{Ni}$ systems [3] (Fig. 1) and $\mathrm{Ca}+\mathrm{Ca}$ systems [17] (Fig. 4). Neither of these
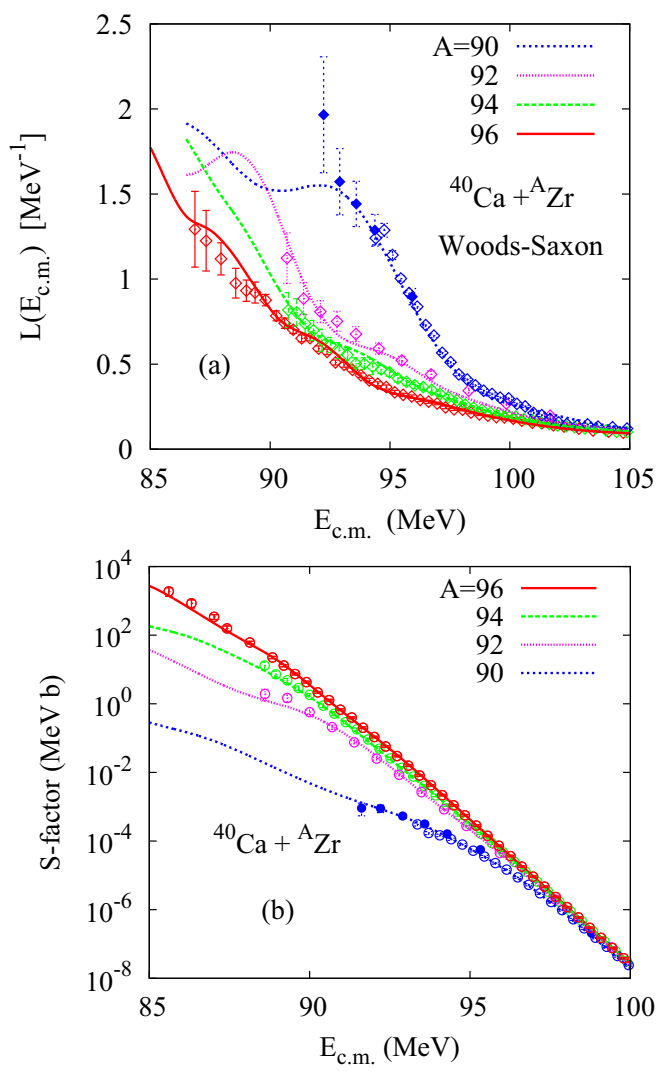

FIG. 4. The logarithmic slopes (a) and the astrophysical $S$ factors (b) for the fusion of the four ${ }^{40} \mathrm{Ca}+{ }^{A} \mathrm{Zr}$ systems $(A=90,92,94$, and 96) are compared to the results of $C C$ calculations. The results were obtained from the measured and calculated cross sections shown in Fig. 2. 
features are needed to improve the fit to the ${ }^{40} \mathrm{Ca}+{ }^{90} \mathrm{Zr}$ fusion data shown in Fig. 2 because the fit is already quite good at low energies whereas the calculation is too low at high energies. It therefore seems unlikely that couplings to transfer reactions would reduce the discrepancy with the data for this system.

\section{SLOPES AND S FACTORS}

The comparison of the $\mathrm{CC}$ calculations and the measured excitation functions for ${ }^{40} \mathrm{Ca}+{ }^{90,92} \mathrm{Zr}$, for which we report here new data, is made in Fig. 1. The calculations show in increasing order the Ch-1 no-coupling limit, the Ch-6 onephonon, the Ch-17 two-phonon, and the Ch-29 three-phonon calculations. In the case of ${ }^{40} \mathrm{Ca}+{ }^{92} \mathrm{Zr}$ we also show the result of the $\mathrm{Ch}-87$ calculation, which also includes the effect of oneand two-nucleon transfer. The parameters for this calculation are indicated by the (*) in Table III. It is seen that the influence of transfer at sub-barrier energies is very large in comparison to the Ch-29 calculation. Moreover, the agreement with the data is surprisingly good in this case. A similar situation was observed for the fusion of ${ }^{40} \mathrm{Ca}+{ }^{96} \mathrm{Zr}$ in Ref. [6].

In order to highlight the sensitivity to the number of channels included in the calculations and, in particular, to the influence of transfer, we show in Figs. 3(a) and 3(b) the logarithmic slopes of the the measured and calculated fusion cross sections for ${ }^{40} \mathrm{Ca}+{ }^{90} \mathrm{Zr}$ and ${ }^{40} \mathrm{Ca}+{ }^{92} \mathrm{Zr}$, respectively. Excellent agreement with the full calculations is achieved in both cases, except at the lowest point for ${ }^{40} \mathrm{Ca}+{ }^{90} \mathrm{Zr}$. In the latter case the logarithmic slope of the Ch-29 calculation saturates, whereas slope of the new data indicated by solid points keeps rising with decreasing energy. This indicates the onset of a fusion hindrance at the lowest energies of the new data, whereas the old data illustrated by the open circles do not show any sign of a hindrance.

The logarithmic slopes for all four ${ }^{40} \mathrm{Ca}+\mathrm{Zr}$ systems are compared in Fig. 4(a) to the slopes of the most realistic calculations. Again it is observed that the largest discrepancy between data and calculations occurs for ${ }^{40} \mathrm{Ca}+{ }^{90} \mathrm{Zr}$, where the measured slope keeps rising at the lowest energy whereas the calculated slope saturates. Since all calculations are based on a standard WS potential, the figure suggests that there is no sign of a fusion hindrance at the lowest energies, except in the fusion of ${ }^{40} \mathrm{Ca}+{ }^{90} \mathrm{Zr}$.

The onset of a hindrance in the fusion of ${ }^{40} \mathrm{Ca}+{ }^{90} \mathrm{Zr}$ is confirmed in Fig. 4(b) where the measured and calculated $\mathrm{S}$ factors for fusion are compared. The $S$ factor is here defined as

$$
S=E_{\mathrm{c} . \mathrm{m} .} \sigma_{f} \exp \left(\eta-\eta_{0}\right),
$$

where $\eta$ is the usual Sommerfeld parameter and $\eta_{0}$ is its value at the reference energy $E_{\text {ref }}=91 \mathrm{MeV}$. It is seen that the $S$ factors keep increasing with decreasing energy for most of the systems, except in the case of ${ }^{40} \mathrm{Ca}+{ }^{90} \mathrm{Zr}$ where the $S$ factor of the lowest two points are identical, i.e., the $S$ factor appears to have reached a maximum.

\section{CONCLUSIONS}

The excitation functions for the fusion of ${ }^{40} \mathrm{Ca}+{ }^{92,90} \mathrm{Zr}$ have been measured from above the barrier down to the level of $\simeq 60$ and $40 \mu \mathrm{b}$, respectively). The previously measured cross sections for ${ }^{40} \mathrm{Ca}+{ }^{90} \mathrm{Zr}$ have been extended downward by a factor $\simeq 20$, while no previous data existed for ${ }^{40} \mathrm{Ca}+{ }^{92} \mathrm{Zr}$.

The experimental results have been analyzed by $\mathrm{CC}$ calculations using a standard Woods-Saxon potential and following the spirit of the previous analyses for ${ }^{40} \mathrm{Ca}+{ }^{96} \mathrm{Zr}$. The calculations give a good account of the new data, as well as of the existing excitation function for ${ }^{40} \mathrm{Ca}+{ }^{94} \mathrm{Zr}$, apart from the persisting underprediction of the high-energy data for ${ }^{40} \mathrm{Ca}+{ }^{90} \mathrm{Zr}$.

Besides one- and multiphonon excitations of the low-lying collective modes, it appears that couplings to transfer channels are very important in the fusion of ${ }^{40} \mathrm{Ca}+{ }^{92} \mathrm{Zr}$ and ${ }^{40} \mathrm{Ca}+$ ${ }^{94} \mathrm{Zr}$, as they were in the fusion of ${ }^{40} \mathrm{Ca}+{ }^{96} \mathrm{Zr}$. The strength of the pair-transfer coupling in these two cases has been deduced by applying a simple recipe based on the number of valence neutrons and the value obtained for ${ }^{40} \mathrm{Ca}+{ }^{96} \mathrm{Zr}$.

Further progress can be made by performing measurements of the relevant one- and two-nucleon transfer reactions, by calibrating the calculations accordingly, and expanding them to include both proton and neutron transfer channels.

In the present work, good agreement is achieved for the low-energy logarithmic slopes in most cases, except at the lowest energies for ${ }^{40} \mathrm{Ca}+{ }^{90} \mathrm{Zr}$, where the experimental slope exceeds the calculated value. This indicates that a fusion hindrance is setting in for this system at the lowest energies, whereas there is no indication of a fusion hindrance for the other three systems. This observation is confirmed by comparing the measured and calculated $S$ factors because a maximum of the $S$ factor is only observed in the data for the system ${ }^{40} \mathrm{Ca}+{ }^{90} \mathrm{Zr}$. The observation is consistent with the interpretation that a fusion hindrance disappears in systems with large positive $Q$ values for transfer $\left({ }^{40} \mathrm{Ca}+{ }^{92,94,96} \mathrm{Zr}\right)$, i.e., in systems where the repulsion due to the Pauli blocking of transfer reactions is absent.

\section{ACKNOWLEDGMENTS}

It is a pleasure to acknowledge the professional work of the XTU Tandem staff, and of M. Loriggiola for preparing targets of excellent quality. The research leading to these results has received funding from the the European Union Seventh Framework Programme FP7/2007-2013 under Grant Agreement No. 262010-ENSAR. P.C., S.S., and N.S. were partially supported by the Croatian Science Foundation under the project 7194. H.E. is supported by the U.S. Department of Energy, Office of Science, Office of Nuclear Physics, Contract No. DE-AC02-06CH11357. A.G. was partially supported by the P210 Excellence Laboratory.
[1] B. B. Back, H. Esbensen, C. L. Jiang, and K. E. Rehm, Rev. Mod. Phys. 86, 317 (2014).
[2] R. A. Broglia, C. H. Dasso, S. Landowne, and A. Winther, Phys. Rev. C 27, 2433 (1983). 
[3] M. Beckerman, M. Salomaa, A. Sperduto, H. Enge, J. Ball, A. DiRienzo, S. Gazes, Y. Chen, J. D. Molitoris, and N.-f. Mao, Phys. Rev. Lett. 45, 1472 (1980).

[4] A. M. Stefanini et al., Phys. Lett. B 728, 639 (2014).

[5] H. Timmers et al., Phys. Lett. B 399, 35 (1997); Nucl. Phys. A 633, 421 (1998).

[6] H. Esbensen, G. Montagnoli, and A. M. Stefanini, Phys. Rev. C 93, 034609 (2016).

[7] L. Corradi, S. Szilner, G. Pollarolo, G. Colò, P. Mason, E. Farnea, E. Fioretto, A. Gadea, F. Haas, D. Jelavić-Malenica, N. Mărginean, C. Michelagnoli, G. Montagnoli, D. Montanari, F. Scarlassara, N. Soić, A. M. Stefanini, C. A. Ur, and J. J. Valiente-Dobón, Phys. Rev. C 84, 034603 (2011).

[8] H. Esbensen and A. M. Stefanini, Phys. Rev. C 89, 044616 (2014).

[9] Ş. Mişicu and H. Esbensen, Phys. Rev. C 75, 034606 (2007).

[10] C. Simenel, A. S. Umar, K. Godbey, M. Dasgupta, and D. J. Hinde, Phys. Rev. C 95, 031601(R) (2017).

[11] A. M. Stefanini, B. R. Behera, S. Beghini, L. Corradi, E. Fioretto, A. Gadea, G. Montagnoli, N. Rowley, F. Scarlassara, S. Szilner, and M. Trotta, Phys. Rev. C 76, 014610 (2007).
[12] A. M. Stefanini, G. Montagnoli, L. Corradi, S. Courtin, E. Fioretto, A. Goasduff, F. Haas, P. Mason, R. Silvestri, P. P. Singh, F. Scarlassara, and S. Szilner, Phys. Rev. C 82, 014614 (2010).

[13] O. Tanimura, Phys. Rev. C 35, 1600(R) (1987).

[14] H. Esbensen, S. Landowne, and C. Price, Phys. Rev. C 36, 1216 (1987).

[15] J. Gomez-Comacho and R. C. Johnson, J. Phys. G 12, L235 (1986).

[16] A. Winther, Nucl. Phys. A 594, 203 (1995).

[17] G. Montagnoli, A. M. Stefanini, C. L. Jiang, H. Esbensen, L. Corradi, S. Courtin, E. Fioretto, A. Goasduff, F. Haas, A. F. Kifle, C. Michelagnoli, D. Montanari, T. Mijatović, K. E. Rehm, R. Silvestri, P. P. Singh, F. Scarlassara, S. Szilner, X. D. Tang, and C. A. Ur, Phys. Rev. C 85, 024607 (2012).

[18] T. Kibédi and R. H. Spear, At. Data Nucl. Data Tables 80, 35 (2002).

[19] ENDSF, NNDS, Brookhaven National Laboratory, www.nndc.bnl.gov/ensdf.

[20] K. Hagino and N. Takigawa, Prog. Theor. Phys. 128, 1061 (2012).

[21] H. Esbensen and C. L. Jiang, Phys. Rev. C 79, 064619 (2009). 